\title{
Evaluation of severity in critically ill patients (CIPS) with limitation of life-sustaining therapy (LLST)
}

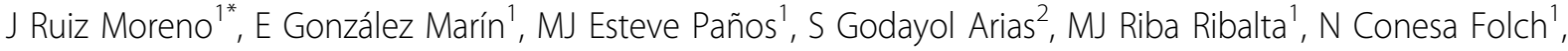 \\ R Corcuera Romero de la Devesa ${ }^{1}$, F Baigorri González ${ }^{1}$, A Artigas Raventós ${ }^{3}$
}

From ESICM LIVES 2015

Berlin, Germany. 3-7 October 2015

\section{Introduction}

It is considered that the severity of CIPs with LLSt is higher than the overall CIPs requiring ICU admission. The identification of specific clinical variables which determine the severity of the CIPs with LLST perhaps has not been researched enough.

\section{Objectives}

- To asses the severity of the CIPs with LLST in comparison to CIPs with LLS.

- To evaluate and compare the mortality between two populations.

\section{Methods}

- Type of study: prospective, analytical, longitudinal, and observational

- Period: January 1-2011 / June 30-2014 (42 months)
- Setting : Medical / Surgical ICU

- Population: 2559 CIPs admitted consecutively to the ICU; sample: 220 CIPs with LLST.

- Exclusión criteria: CIPs $<16$ y., major burn CIPs, incomplete clinical documentation, and voluntary discharge.

- Variables analyzed:

\section{a) Age}

b) Hospital mortality

c) Case - mix: severe sepsis, metabolic acidosis, total parenteral nutrition (TPN), oncological pathology, intraabdominal pressure (IAP), blood products, cultures, cardiac continous output (CCO), advanced life support (ALS) before LLTS applied, FGC, FBC.

- Statistical analysis: Ji squared and contrast of means (Student's t)

Table 1. Results 1

\begin{tabular}{llllllll}
\hline & Global & \% - SD & LLST & \% - SD & without LLST & \% - SD & p value \\
\hline CIPs & 2559 & 100 & 220 & 8,6 & 2339 & 91,4 & 0,0001 \\
\hline Age & 65,9 & 16,7 & 77,2 & 10,6 & 64,8 & 16,8 & 0,0001 \\
\hline Mortality & 182 & 7,1 & 115 & 52,3 & 67 & 2,96 & 0,0001 \\
\hline Sepsis & 484 & 18,9 & 122 & 55,4 & 362 & 15,4 & 0,0001 \\
\hline Metabolic acid. & 955 & 37,3 & 155 & 70,4 & 800 & 34,2 & 0,0001 \\
\hline oncol. pathology & 903 & 35,3 & 84 & 38,2 & 819 & 35,0 & NS \\
\hline TPN & 467 & 18,27 & 72 & 32,7 & 395 & 16,8 & 0,0001 \\
\hline
\end{tabular}


Table 2. Results II

\begin{tabular}{|c|c|c|c|c|c|c|c|}
\hline & Global & $\%-S D$ & LLST & $\%-S D$ & without LLST & $\%-S D$ & $p$ value \\
\hline IAP & 136 & 5,3 & 51 & 23,1 & 85 & 3,63 & 0,0001 \\
\hline Blood products & 500 & 19,5 & 99 & 45,0 & 401 & 17,1 & 0,0001 \\
\hline Cultures & 689 & 26,9 & 144 & 65,4 & 545 & 23,3 & 0,0001 \\
\hline ALS & 85 & 3,32 & 16 & 7,3 & 69 & 2,9 & 0,0001 \\
\hline FGC & 54 & 2,1 & 21 & 9,5 & 33 & 1,4 & 0,0001 \\
\hline $\mathrm{FBC}$ & 61 & 2,4 & 24 & 10,9 & 37 & 1,6 & 0,0001 \\
\hline $\mathrm{CCO}$ & 114 & 4,4 & 46 & 20,9 & 68 & 2,9 & 0,0001 \\
\hline
\end{tabular}

\section{Results}

See tables 1 and 2 .

\section{Conclusions}

- More than $50 \%$ of CIPs with LLST die.

- According to all clinical variables, SS, metabolic acidosis, TPN, IAP, blood products, cultures, ALS, FGC, FBC, and cardiac output are much higher in CIPs with LLST.

\section{Authors' details}

'QuirónSalud Hospital Universitario Sagrat Cor, Critical Care Department, Barcelona, Spain. ${ }^{2}$ QuirónSalud Hospital Universitario Sagrat Cor, Emergency Department, Barcelona, Spain. ${ }^{3}$ Hospital de Clínicas de Sabadell \&

QuirónSalud Hospital Universitario Sagrat Cor, Critical Care Department,

Sabadell, Spain.

Published: 1 October 2015

\section{References}

1. Wiegand DL, Grant MS: Bioethical Issues Related to Limiting Lifesustaining Therapies in the Intensive Care Unit Disclosures. J Hospice Palliative Nursing 2014, 16(2):60-64.

2. Wunsch $\mathrm{H}$, Harrison DA, Harvey S, Rowan K: End of life decisions: a cohort study of the withdrawl of all active treatment in intensive care units in the United Kingdom. Intensive Care Med 2005, 31:823-31.

Cite this article as: Ruiz Moreno et al:: Evaluation of severity in critically ill patients (CIPS) with limitation of life-sustaining therapy (LLST).

Intensive Care Medicine Experimental 2015 3(Suppl 1):A659.

\section{Submit your manuscript to a SpringerOpen ${ }^{\mathcal{O}}$ journal and benefit from:}

- Convenient online submission

- Rigorous peer review

- Immediate publication on acceptance

- Open access: articles freely available online

- High visibility within the field

- Retaining the copyright to your article 